\title{
Algorithms for Designing Clamshell Molds
}

\author{
Stefanie Wuhrer ${ }^{1}$, Prosenjit Bose ${ }^{1}$, Pat Morin ${ }^{1}$ and Michiel Smid ${ }^{1}$ \\ ${ }^{1}$ Carleton University, \{swuhrer, jit, morin, michiel $\} @$ scs.carleton.ca
}

\begin{abstract}
Clamshell casting is a popular manufacturing technique where liquid is poured into a mold or cast and the cast is removed once the liquid has hardened. The term clamshell refers to the way in which the cast is removed. The two cast parts are removed by rotation through a hinge much like a clamshell. Given an object, modeled by a polyhedron $P$ of arbitrary genus with combinatorial complexity $n$, we determine when the surface of the polyhedron can be decomposed into two parts s.t. each part can be rotated around a line in space without colliding with each other or intersecting the interior. Such a line is a valid casting line. Specifically, we solve the following problems: (1) Given a line $l$ in space, we determine in $O(n)$ time whether $l$ is a valid casting line for $P$. If degeneracies are present, such as a reflex edge or face of $P$ is perpendicular to $l$, the running time increases slightly to $O(n \log n)$. (2) In $O\left(n^{4} \alpha(n)\right)$ time, where $\alpha(n)$ is the inverse Ackermann function, we report all combinatorially distinct valid casting lines. The running time is $O\left(n^{4} \log n\right)$ in the presence of degeneracies. All of the running times are shown to be almost optimal.
\end{abstract}

Keywords: Computational geometry, theoretical foundations of CAD, casting, manufacturing.

\section{INTRODUCTION}

The problem of determining whether a given object modeled by a polyhedron can be manufactured using the casting process is a well-studied problem in computational geometry (see [2],[6],[8],[10] for various surveys of the area). Most of the research has concentrated on determining whether the surface of a polyhedron with combinatorial complexity $n$ can be partitioned into two pieces s.t. the polyhedron's boundary can be removed by translations (referred to as translational casting). Removal of these two pieces of the surface of a polyhedron by translation naturally models several known casting processes.

Bose et al. [3] considered objects modeled by a simple $n$-vertex polyhedron. They provided an $O\left(n^{2} \log n\right)$ time algorithm to determine if a plane can partition the surface of a polyhedron into two pieces s.t. each piece can be removed by a translation. The running time of the algorithm can be improved to $O\left(n^{3 / 2+\varepsilon}\right)$, for any fixed $\varepsilon>0$, by using more complicated data structures. Furthermore, the running time of the algorithm becomes $O\left(n^{2}\right)$ in case that the cast removal directions are opposite directions.

Ahn et al. [1] generalized these results by removing the constraint that the polyhedron is partitioned with a plane and by studying polyhedra of arbitrary genus. Given a direction $d$, they provide an $O(n \log n)$ time algorithm for determining if the surface of a polyhedron with combinatorial complexity $n$ can be partitioned into exactly two pieces s.t. one piece can be translated in direction $d$ and the other in direction $-d$. They also provide an $O\left(n^{4}\right)$ time algorithm to determine all valid casting directions for a given polyhedron. Note that the part of the surface of $P$ that is translated is a terrain. Fekete and Mitchell [7] showed that decomposing the boundary of a simple polyhedron into the minimum number of terrains is NP-hard.

In this paper, we initiate the study of clamshell casting (or rotational casting). Informally, in this manufacturing process, one can imagine that the cast for a given object is a two-piece 'shell' with the pieces attached by a hinge. Liquid is poured into the cast and once the liquid has hardened, the shell is opened to allow removal of the object. The shell is opened by having each of the two pieces of the shell rotate around the hinge. Hence, the ultimate goal of 
the study of clamshell casting is to determine for any given object whether there exists a clamshell cast that can be opened by a rotation around the hinge, s.t. the object can be removed from the cast without breaking the object or the cast. This paper takes a first step towards this ultimate solution and presents algorithms to determine whether there exists a clamshell cast that can be opened by a rotation around the hinge for polyhedral objects. However, further research is required to examine removing the object from the clamshell cast without breaking the object or the cast. Hence, the conditions on clamshell casting presented in this paper are necessary but not sufficient.

We model this problem mathematically as follows. The object is modeled by a polyhedron $P$ of arbitrary genus with combinatorial complexity $n$. The boundary of $P$ is the cast of $P$. The hinge is modeled by a line $l$ in 3 -space. The first problem we address is given a line of rotation $l$, determine whether there exists a partitioning of the cast into exactly two parts, s.t. one part can be rotated clockwise $(\mathrm{cw})$ around $l$ and the other part can be rotated counterclockwise (ccw) around $l$ without colliding with the interior of $P$ or the cast. Such a line is called a valid casting line. We provide an $O(n)$ time to determine if a given line is a valid casting line. If certain degeneracies are present, such as a reflex edge or face of $P$ is perpendicular to $l$, the running time increases slightly to $O(n \log n)$. These degeneracies increase the complexity because they introduce ambiguity. If a face is perpendicular to $l$, then it can be removed both in $\mathrm{cw}$ and ccw orientation around $l$. The extra complexity is needed to resolve this ambiguity.

Next, we address the problem of finding all valid casting lines for $P$. In $O\left(n^{4} \alpha(n)\right)$ time, where $\alpha(n)$ is the inverse Ackermann function, we report all lines that allow a valid cast partitioning. Again, in the presence of degeneracies, the running time is slightly worse: $O\left(n^{4} \log n\right)$. We provide an example of a class of polyhedra with $\Omega\left(n^{4}\right)$ combinatorially distinct valid casting lines. Hence, the algorithms we present are almost worst case optimal.

Since translational casting can be considered as rotational casting with the line of rotation placed at infinity, one suspects that the two are related. In fact, there exists a close relationship between rotational casting and translational casting. Let $P$ denote a polyhedron and let $l$ denote the axis of rotation. Assume that $P$ and $l$ are given in a cylindrical coordinate system with $l$ as $z$-axis. Transform the coordinate system into a Cartesian coordinate system, s.t. the $x$-axis describes the angle $\varphi$, the $y$-axis describes the distance $d$, and the $z$-axis stays fixed. Considering this transformed system between $x=0$ and $x=2 \pi$ shows the transformed cylindrical coordinate system. Every point of the cast of $P$ moves along a straight line when the cast is removed. Hence, rotational casting becomes translational casting. It remains to analyze the shape of the transformed polyhedron $\tilde{P}$. Assume that $P$ does not contain vertical edges or faces and that $l$ does not intersect the interior of. The transformation of a face $f$ of $P$ is in essence a curve describing the distance of points on the face to the origin and can be described using trigonometric functions. The transformed polyhedron $\tilde{P}$ is topologically equivalent to $P$ and its boundary consists of piecewise non-algebraic surface patches. Considering rotational casting of a polyhedron $P$ is equivalent to considering translational casting of the transformed polyhedron $\tilde{P}$. Unfortunately, none of the known algorithms for translational casting can be easily extended to handle translational castability of three-dimensional objects bounded by piecewise non-algebraic surface patches. Thus, we were unable to use any of the tools and techniques developed for translational casting.

As this is an extended abstract, most proofs are omitted due to space constraints. All proofs are available in the full version of the paper [4].

\section{PRELIMINARIES}

Define a polyhedron $P$ of arbitrary genus as a closed, compact, connected subset of $\mathbb{R}^{3}$ bounded by a piecewise linear surface. Let $\operatorname{int}(P)$ and $\partial P$ denote the interior and boundary of $P$, respectively, so that $P=\operatorname{int}(P) \cup \partial P$.

The boundary is also called the cast of $P$. Two faces are adjacent if they share at least one edge. Parallel adjacent faces are not allowed, since this can be easily avoided by merging the two adjacent parallel faces. Let $n$ denote the combinatorial complexity of $P$ (the combinatorial complexity of $P$ is the sum of the number of all the edges, vertices, and faces of $P$ ). The aim is to rotationally remove the cast of $P$ in two pieces.

Definition 1. Let $l$ be a directed line in three-space. Consider the plane $\pi$ perpendicular to $l$ passing through a point $p$ of $P$. Denote the intersection point of $\pi$ and $l$ by $l^{\prime}(p)$. Denote the circular arc with center $l^{\prime}(p)$ and angle

Computer-Aided Design \& Applications, Vol. 4, Nos. 1-4, 2007, pp 1-10 
$\alpha$ starting at $p$ winding in clockwise $(\mathrm{cw})$ or counterclockwise (ccw) direction by $\operatorname{cwarc}\left(l^{\prime}(p), p, \alpha\right)$ or $\operatorname{ccwarc}\left(l^{\prime}(p), p, \alpha\right)$ respectively. A face $f$ of $P$ is called removable in $c w$ orientation w.r.t. $l$ if $\exists \alpha>0$ s.t. $\forall p$ on $f \operatorname{cwarc}\left(l^{\prime}(p), p, \alpha\right) \cap \operatorname{int}(P)=\varnothing$ and removable in $c c w$ orientation w.r.t. $\quad l \quad$ if $\quad \exists \alpha>0 \quad$ s.t. $\quad \forall p \quad$ on $f \operatorname{ccwarc}\left(l^{\prime}(p), p, \alpha\right) \cap \operatorname{int}(P)=\varnothing$. The $\mathrm{cw}$ and ccw orientation is measured w.r.t. the orientation of line $l$.

The $\mathrm{cw}$ or ccw orientation is then called a valid orientation for cast removal for $f$ w.r.t. $l$ respectively, and $l$ is called a valid casting line for $f$. The validity extends to the validity of a polyhedron as follows:

Definition 2. Let $l$ be a directed line. A polyhedron $P$ is rotationally castable w.r.t. $l$, if $\partial P$ can be partitioned into exactly two non-empty connected components $P_{1}$ and $P_{2}$, s.t. all faces of $P_{1}$ are removable in cw orientation w.r.t. $l$, all faces of $P_{2}$ are removable in ccw orientation w.r.t. $l, \exists \alpha>0$ s.t. $\forall p \in P_{1} \operatorname{cwarc}\left(l^{\prime}(p), p, \alpha\right) \cap \operatorname{int}\left(P_{2}\right)=\varnothing$, and $\exists \alpha>0$ s.t. $\forall p \in P_{2} \operatorname{ccwarc}\left(l^{\prime}(p), p, \alpha\right) \cap \operatorname{int}\left(P_{1}\right)=\varnothing$.

The last two conditions of the definition ensure that the two components $P_{1}$ and $P_{2}$ do not obstruct each other's rotational paths. Fig. 1 shows part of a simple polyhedron that is decomposed into $P_{1}$ and $P_{2}$. Although $\partial P$ can be decomposed into $P_{1}$ and $P_{2}$, s.t. $P_{1}$ is removable in cw orientation and $P_{2}$ is removable in ccw orientation, the two components cannot be rotated by any angle, because they obstruct each other. In the following, the notations rotationally castable and castable are used interchangeably. Note that the partitioning of the polyhedron is not necessarily at edges of $P$.

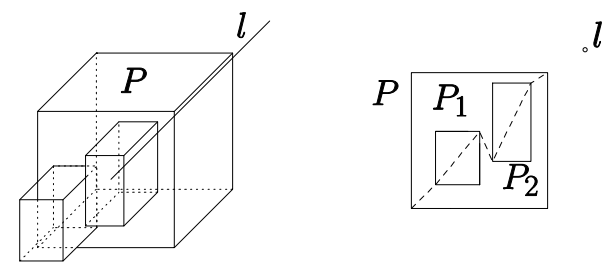

Fig. 1: Two different views of $P$ are shown along with the partitioning of $P$. The components $P_{1}$ and $P_{2}$ obstruct each other's rotational paths.

Certain degeneracies make the determination of castability difficult. In particular when a face or reflex edge of $P$ is perpendicular to the casting line. Thus, we say that $P$ is robustly castable w.r.t. a line $l$ provided that it is castable w.r.t. $l$ and no face or reflex edge of $P$ is perpendicular to $l$. A line that is perpendicular to a face or reflex edge of $P$ will be called a degenerate line.

\section{DECISION PROBLEM}

In this section, we address the problem of determining whether a polyhedron $P$ with combinatorial complexity $n$ is robustly castable with respect to a given line $l$ of rotation and present an algorithm that solves the problem in $O(n)$ time. In the presence of degeneracies, the algorithm takes $O(n \log n)$ time.

We first address the problem of robust castability w.r.t. a line $l$. The problem can be decomposed into three subproblems: determining the valid orientation for cast removal for all faces of $P$ w.r.t. $l$, checking whether all the faces removable in $\mathrm{cw}$ and $\mathrm{ccw}$ orientation w.r.t. $l$ form a connected component of $P$, respectively, and testing whether the two components can be rotated around $l$ without colliding. We address each subproblem in order.

Definition 3. Let $f$ be a face of $P$ and denote its inner normal by $\vec{n}$. Define the open unbounded prism $S(f)=\{p+t \vec{n}: p \in f, t \in \mathbb{R}\}$. Denote $S^{+}(f)=\{p+t \vec{n}: p \in f, t>0\}, S^{-}(f)=\{p+t \vec{n}: p \in f, t \leq 0\}$.

The prisms of the definition are illustrated in Fig. 2. 


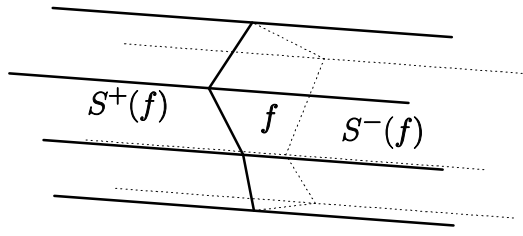

Fig. 2: The prisms $S^{+}(f)$ and $S^{-}(f)$.

In a first step, the algorithm determines whether any reflex edge or face of $P$ is perpendicular to the given line $l$. This test takes constant time per reflex edge or face, respectively, and therefore $O(n)$ total time. If any reflex edge or face of $P$ is perpendicular to $l, P$ is not robustly castable w.r.t. $l$ by definition. Otherwise, we determine the valid orientation for cast removal for each face of $P$. In the following, we can therefore assume that no reflex edge or face of $P$ is perpendicular to $l$.

Let $f$ be a face of $P$ with inner normal $\vec{n}$. If $l \cap S(f)=\varnothing$, compute a point $p \in \partial f$ that minimizes the Euclidean distance between $\partial f$ and $l$. If $p$ is not unique, the set of points on $\partial f$ with minimal distance from $l$ are located on an edge of $\partial f$ parallel to $l$ and an arbitrary point $p$ of that set is picked. Denote by $\tau$ the plane with normal $\vec{n} \times \vec{l}$ passing through $p$ and by $p^{*}$ the point $p$ translated by $\vec{n} \times \vec{l}$. Denote the open half space induced by $\tau$ containing $p^{*}$ by $\tau^{+}$and the open half space induced by $\tau$ not containing $p^{*}$ by $\tau^{-}$. The following lemma characterizes all locations from which a face is removable.

Lemma 1. For the orientation for cast removal of $f$, the following four cases are possible:

1. $f$ is removable from the cast using only a cw orientation around $l$, iff $l \cap S(f)=\varnothing$ and $l \in c l\left(\tau^{-}\right)$.

2. $f$ is removable from the cast using only a ccw orientation around $l$, iff $l \cap S(f)=\varnothing$ and $l \in c l\left(\tau^{+}\right)$.

3. $f$ needs to be partitioned into two or more parts along the orthogonal projection of $l$ on $f$, iff $l \cap S^{+}(f)=\varnothing$ and $l \cap S^{-}(f) \neq \varnothing$. One or more parts of $f$ are removable using a ccw rotation and the other ones using a cw rotation around $l$.

4. $\quad f$ is not removable from the cast, iff $l \cap S^{+}(f) \neq \varnothing$.

Lemma 1 classifies all faces of $P$ into four categories. In linear time, all the faces of $P$ can be categorized. If any face of $P$ is not removable w.r.t. $l, P$ is not castable w.r.t. $l$. Otherwise, the next step is to determine if $\partial P$ can be decomposed into two connected components. In particular, the faces of $P$ that need to be split (faces of type 3 in Lemma 1) need to be examined more carefully as outlined in the following lemma.

Lemma 2. All the faces $f$ of $P$ with $l \cap S^{+}(f)=\varnothing$ and $l \cap S^{-}(f) \neq \varnothing$ can be partitioned along the orthogonal projection of $l$ on $f$ and labeled as removable in cw or ccw orientation w.r.t. $l$ respectively in $O(n)$ time.

Every face $f$ of $P$ with $l \cap S^{+}(f)=\varnothing$ and $l \cap S^{-}(f) \neq \varnothing$ is split along the orthogonal projection of $l$ on $f$. The number of new split edges that are inserted is $O(n)$. This yields the conclusion that all the faces of $P$ are labeled as removable in $\mathrm{cw}$ or $\mathrm{ccw}$ orientation w.r.t. $l$ in $O(n)$ time.

The next step is to determine if the labels on the faces admit a decomposition of $\partial P$ into two connected components. $\mathrm{By}$ applying depth-first search on the set of faces with a particular label, one can determine connectivity in linear time. We summarize this in the following lemma.

Computer-Aided Design \& Applications, Vol. 4, Nos. 1-4, 2007, pp 1-10 
Lemma 3. Given a partitioning of $\partial P$ into two components $P_{1}$ and $P_{2}$, s.t. all faces in $P_{1}$ are removable in cw orientation w.r.t. $l$ and all faces in $P_{2}$ are removable in ccw orientation w.r.t. $l$, we can determine whether $P_{1}$ and $P_{2}$ are connected in time $O(n)$.

The final step is to determine whether $P_{1}$ can be rotated in $\mathrm{cw}$ orientation w.r.t. $l$ without colliding with $P_{2}$ and whether $P_{2}$ can be rotated in ccw orientation w.r.t. $l$ without colliding with $P_{1}$. The fact that the rotations of $P_{1}$ and $P_{2}$ do not collide with the interior of $P$ has been established by the validity of each face.

Lemma 4. Given a partitioning of $P$ into connected components $P_{1}$ and $P_{2}$, s.t. all faces in $P_{1}$ are removable in $\mathrm{cw}$ orientation w.r.t. $l$ and all faces in $P_{2}$ are removable in ccw orientation w.r.t. $l$, we can test whether $\exists \alpha>0$ s.t. $\forall q$ on $P_{1} \operatorname{cwarc}\left(l^{\prime}(q), q, \alpha\right) \cap P_{2}=\varnothing$ (equivalently $\exists \alpha>0$ s.t. $\forall q$ on $\left.P_{2} \operatorname{ccwarc}\left(l^{\prime}(q), q, \alpha\right) \cap P_{1}=\varnothing\right)$ in time $O(n)$.

The proof of this lemma extends results by Bose et al. [5] for rotational casting in two dimensions and can be found in [4]. This yields the following result:

Theorem 1. Given a polyhedron $P$ with combinatorial complexity $n$ and a line of rotation $l$, it is possible to determine whether $P$ is robustly castable w.r.t. $l$ in time $O(n)$.

Now, we turn our attention to general rotational casting. Recall that the only difference between a polyhedron being robustly rotationally castable and rotationally castable is the degenerate situation where faces or reflex edges of $P$ are perpendicular to $l$. We outline how to handle this situation which turns out to impose an additional $\log n$ factor on the running time. Thus, determining whether a polyhedron $P$ is castable w.r.t. $l$ takes $O(n \log n)$ time.

Consider the notation of Lemma 1 . In the case of general castability of $P$, it is possible that a face $f$ of $P$ with inner normal $\vec{n}$ is perpendicular to the given line $l$.

Observation 1. Let $f$ be a face of $P$ with inner normal $\vec{n}$ and let $l$ be a line in direction $\vec{l}$. If $l \cap S(f)=\varnothing$ and $\vec{n} \times \vec{l}=\overrightarrow{0}, f$ is removable in both cw and ccw orientation w.r.t. $l$.

After labeling all the faces as before, it remains to split the faces with $l \cap S(f)=\varnothing$ and $\vec{n} \times \vec{l}=\overrightarrow{0}$ and assign unique valid orientations to the newly created faces if possible. The face $f$ can be partitioned into two (possibly empty) sets of faces $F_{1}$ and $F_{2}$, s.t. all the faces adjacent to $f$ removable in cw orientation w.r.t. $l$ are adjacent to faces in $F_{1}$ and all the faces adjacent to $f$ removable in $\mathrm{ccw}$ orientation w.r.t. $l$ are adjacent to faces in $F_{2}$. If there exists a partitioning of $f$ into $F_{1}$ and $F_{2}$ s.t. $f$ is removable w.r.t. $l$, we call the partitioning a valid partitioning of $f$.

If $l$ passes through a hole of a face $f$ perpendicular to $l$, there are two possible cases: $l \cap \partial P \neq \varnothing$ and $l \cap \partial P=\varnothing$. If $l \cap \partial P \neq \varnothing$, at least one of the faces of $P$ intersects $l$ and is therefore not removable w.r.t. $l$. If $l \cap \partial P=\varnothing$, the boundary of the hole of $P$ that contains $l$ cannot be removed without penetrating $\operatorname{int}(P)$.

Lemma 5. For all faces $f$ of $P$ with inner normal $\vec{n}, l \cap \partial P=\varnothing$, and $\vec{l}$ is parallel to $\vec{n}$, we can find a valid partitioning or report that $f$ is not removable w.r.t. $l$ in total time $O(n \log n)$.

This yields the conclusion that all the faces of a polyhedron $P$ with combinatorial complexity $n$ can be labeled as removable in $\mathrm{cw}, \mathrm{ccw}$, or no orientation w.r.t. a line of rotation $l$ in space in $O(n \log n)$ total time. Since Lemmata 3 and 4 still hold in the general setting, we conclude with the following theorem:

Theorem 2. Given a polyhedron $P$ with combinatorial complexity $n$ and a line of rotation $l$, it is possible to determine whether $P$ is castable w.r.t. $l$ in time $O(n \log n)$. 


\section{DETERMINING ALL VALID CASTING LINES}

In this section, we solve the problem of finding all lines $l$, s.t. a given polyhedron is robustly castable with respect to $l$. Recall that a polyhedron $P$ is robustly castable with respect to a line $l$ provided that $P$ is castable w.r.t. $l$ and $l$ is not degenerate. Hence, until Section 4.2, we will assume that all lines considered are not degenerate. We handle degeneracies specifically in Section 4.2. We consider the problem of reporting a representative line for each class of combinatorially distinct robust valid casting line for a given polyhedron. Two valid casting lines $l_{1}$ and $l_{2}$ are combinatorially distinct if $l_{1}$ cannot continuously be moved to $l_{2}$ without becoming invalid.

To solve this problem, we exploit the strong relation between rotational castability in three dimensions and rotational castability in two dimensions. In two dimensions, a collection of polygons $S$ is rotationally castable w.r.t. a point $r$ provided that the boundaries of the polygons can be partitioned into two sets of polygonal chains where one set can be rotated $\mathrm{cw}$ w.r.t. $r$ and the other $\mathrm{ccw}$ w.r.t. $r$ by some angle $\alpha>0$ without colliding with each other or intersecting the interiors of the polygons. The following lemma reduces the three-dimensional casting problem to the twodimensional version studied by Bose et al [5].

Lemma 6. A polyhedron $P$ is robustly castable w.r.t. a line $l$ iff every cross section $P^{\prime}$ of $P$ with a plane $\pi$ perpendicular to $l$ is castable w.r.t. the point $l^{\prime}=\pi \cap l$.

To prove that $P$ is robustly castable w.r.t. $l$ if every cross section $P^{\prime}$ is castable w.r.t. the point $l^{\prime}$, a plane perpendicular to $l$ is swept over $P$ and the topological changes that occur in the cross section $P^{\prime}$ are analyzed. The proof of the lemma proceeds by induction on the genus $\mathrm{g}$ of $\partial P$.

For the two-dimensional casting problem, Bose et al. [5] define black regions for edges and reflex vertices of a simple polygon $S$. A black region for an edge is the set of points for which the edge cannot be rotated in $\mathrm{cw}$ or $\mathrm{ccw}$ direction without penetrating the interior of $S$. Therefore, no point in a black region is a valid casting point for polygon $S$.

Definition 4. Let $e$ be an edge of a simple polygon with vertices $a$ and $b$ and denote the inner normal of $e$ by $\vec{n}(e)$. The open strip $\{\vec{p}+t \vec{n}(e): p \in e \backslash\{a, b\}, t \geq 0\}$ is the black region of $e$.

Let $v$ be a reflex vertex of a simple polygon. Denote the two edges adjacent to $v$ by $e_{1}$ and $e_{2}$ and denote their inner normals by $\vec{n}\left(e_{1}\right)$ and $\vec{n}\left(e_{2}\right)$ respectively. The near cone of $v$ is defined as $\left\{\vec{v}+t_{1} \vec{n}\left(e_{1}\right)+t_{2} \vec{n}\left(e_{2}\right): t_{1}, t_{2} \geq 0, t_{1} t_{2} \neq 0\right\}$ and the far cone of $v$ is defined as $\left\{\vec{v}+t_{1} \vec{n}\left(e_{1}\right)+t_{2} \vec{n}\left(e_{2}\right): t_{1}, t_{2}<0\right\}$. The black region of $v$ is the union of the near cone and the far cone of $v$.

If the center of rotation $r$ is located in the black region of an edge $e, e$ is not removable w.r.t. $r$. Similarly, if $r$ is located in the black region of a reflex vertex $v, v$ cannot be rotated by any angle around $r$ without penetrating the interior of the polygon. Bose et al. [5] prove that a polygon is castable w.r.t. a point $r$ in the plane if and only if $r$ is not contained in the black region induced by edges and reflex vertices of the polygon.

Analogous to the two-dimensional casting problem discussed by Bose et al. [5], we define black regions for faces, reflex edges, and reflex vertices of a polyhedron. These regions will have the property that any line intersecting a black region cannot be a valid casting line.

Definition 5. For a face $f$ of $P$, the black region of $f$ is defined as the set $S^{+}(f)$.

Note that there is no valid casting line for $f$ that properly intersects the black region of $f$ (see Lemma 1). Fig. 2 shows the black region of $f$, which is the prism that is swept when translating $f$ along its inner normal vector.

Definition 6. Let $e$ be a reflex edge of $P$ and denote the inner normals of its two adjacent faces by $\vec{n}_{1}$ and $\vec{n}_{2}$. Define the near wedge of $e$ as $S^{+}(e)=\left\{\vec{p}+t_{1} \vec{n}_{1}+t_{2} \vec{n}_{2}: p \in e, t_{1}, t_{2} \geq 0\right\}$ and the far wedge of $e$ as $S^{-}(e)=\left\{\vec{p}+t_{1} \vec{n}_{1}+t_{2} \vec{n}_{2}: p \in e, t_{1}, t_{2}<0\right\}$. The black region of a reflex edge $e$ is defined as $\left(S^{+}(e) \cup S^{-}(e)\right) \backslash e$. 
Definition 7. A vertex $v$ of $P$ is a reflex vertex of $P$ iff all of $v$ 's adjacent edges are reflex edges of $P$. Let $v$ be a

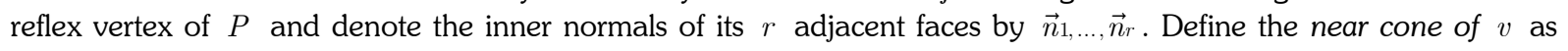
$S^{+}(v)=\left\{\vec{v}+\sum_{i=1}^{r} t_{i} \vec{n}_{i}: t_{i}>0 \forall i=1, \ldots, r\right\}$ and the far cone of $v$ as $S^{-}(v)=\left\{\vec{v}+\sum_{i=1}^{r} t_{i} \vec{n}_{i}: t_{i}<0 \forall i=1, \ldots, r\right\}$. The black region of a reflex vertex $v$ is defined as $S^{+}(v) \cup S^{-}(v)$.

Fig. 3 illustrates the black region of a reflex edge and of a reflex vertex.
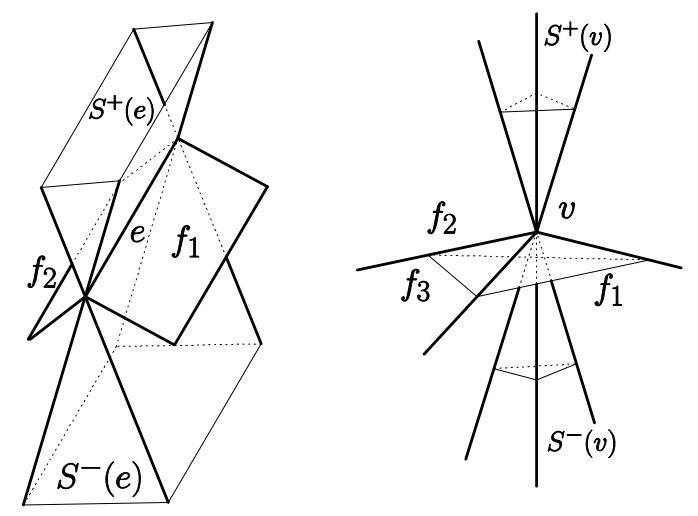

Fig. 3: Left: The near and far wedge of $e$. Right: The near and far cone of $v$.

The black regions of faces, reflex edges, and reflex vertices of $P$ are the black regions induced by $P$.

Lemma 7. A polyhedron $P$ is robustly castable w.r.t. a non-degenerate line $l$, iff $l$ does not intersect any of the black regions induced by $P$.

To prove this statement, we show that $l$ intersects the black regions induced by $P$ iff there exists a cross section of $P$ with a plane $\pi$ perpendicular to $l$, s.t. the point $l^{\prime}=l \cap \pi$ is contained in the black regions induced by $P^{\prime}=P \cap \pi$. The results by Bose et al. [5] and Lemma 6 directly yield the statement of the lemma.

Consider all the planes bounding black regions of faces, reflex edges, and reflex vertices of $P$. The black region of a face $f$ of $P$ is bounded by $\operatorname{deg}(f)$ planes and can be expressed using $\operatorname{deg}(f)$ lines perpendicular to $f$ passing through vertices of $f$. The black region of a reflex edge $e$ of $P$ can be expressed using five lines: four lines perpendicular to the faces adjacent to $e$ passing through the vertices of $e$ and the supporting line of $e$. Finally, the black region of a reflex vertex $v$ of $P$ can be expressed using $\operatorname{deg}(v)$ lines perpendicular to the faces adjacent to $v$ passing through $v$. Define the arrangement $A$ in $\mathbb{R}^{3}$ induced by these $O(n)$ lines. Two distinct lines $g_{1}, g_{2} \in A$ are in the same equivalence class of $A$ iff it is possible to move $g_{1}$ to $g_{2}$ without crossing any of the lines in $A$. If $g_{1}$ does not cross any of the lines in $A$ during the transformation, it does not enter or leave any of the black regions induced by $P$. Assume that $g_{1}$ and $g_{2}$ are in the same equivalence class of $A$. Lemma 7 ensures that $g_{2}$ is a valid casting line for $P$ iff $g_{1}$ is a valid casting line for $P$.

\subsection{Reporting all Valid Casting Lines}

In this section, we show how to find and report a representative for each class of the combinatorially distinct valid robust casting lines for polyhedron $P$ in time $O\left(n^{4} \alpha(n)\right)$, where $\alpha(n)$ is the inverse Ackermann function.

To report all of the valid casting lines for $P$ in $\mathbb{R}^{3}$, we construct the arrangement $A$ of the lines defined as intersections of the planes bounding black regions induced by $P$. Every cell of $A$ corresponds to exactly one 
equivalence class of $A$. Therefore, it is possible to label each cell $c$ of $A$ as an equivalence class of valid or invalid casting lines for $P$. Finally, a representative line for each equivalence class labeled as valid is reported.

The arrangement $A$ of $O(n)$ lines is constructed using an algorithm by McKenna and O'Rourke [9]. They represent $k$ lines in $\mathbb{R}^{3}$ using four parameters per line and construct $O\left(k^{2}\right)$ planar arrangements of hyperbolas in $O\left(k^{2} \alpha(k)\right)$ time each. They show that the arrangement has complexity $\Theta\left(k^{4}\right)$ and can be constructed in time $O\left(k^{4} \alpha(k)\right)$. The arrangement is represented as a graph $G$, where every line touching four of the $k$ given lines or parallel to one of the $k$ given lines and touching two of the remaining $k-1$ given lines is represented as a node of $G$. Using this method, $A$ has complexity $O\left(n^{4}\right)$ and can be constructed in time $O\left(n^{4} \alpha(n)\right)$.

Each node of $G$ represents a combinatorially distinct class of lines and each combinatorially distinct class is represented by a node of $G$. Thus, once $A$ and $G$ are constructed, every node of $G$ is labeled as valid or invalid by traversing the graph $G$. Since a representative line can be tested for validity in linear time, a naive traversal of $G$ would cost $O\left(n^{5}\right)$, however, after determining the validity of one node of $G$, we can take advantage of the coherence between vertices of $G$ to process each additional vertex in constant time. For a detailed explanation of this process, refer to Bose et al. [4]. Therefore, the cost of reporting all valid lines for robust casting is dominated by the time to construct $A$ and $G$. We conclude with the following.

Theorem 3. Given a polyhedron $P$ with combinatorial complexity $n$, it is possible to report all of the valid robust casting lines for $P$ in $\mathbb{R}^{3}$ in time $O\left(n^{4} \alpha(n)\right)$, where $\alpha(n)$ is the inverse Ackermann function.

\subsection{Handling Degeneracies}

The two degenerate situations we address are when a line is perpendicular to a face of $P$ or a reflex edge of $P$. The main problem to overcome is that Lemma 7 does not hold any more in the presence of these degeneracies. There are two reasons for this. First, it is possible for $P$ to be castable w.r.t. $l$ when $l$ intersects a black region arising from one of the degeneracies. However, the only case when $P$ is still castable is if $l$ intersects the far wedge of a reflex edge $e$ of $P$ perpendicular to $l$. In this case, $P$ is castable w.r.t. $l$, because $e$ can be split on the perpendicular projection of $l$ on $e$ and one part of $e$ is removable in $\mathrm{cw}$ orientation w.r.t. $l$ and the other part of $e$ is removable in ccw orientation w.r.t. $l$. Second, there exist lines of rotation $l$, s.t. $l$ does not intersect any of the black regions induced by $P$ but $P$ is not castable w.r.t. $l$. This can only occur, if $P$ contains a face perpendicular to $l$ that is not removable w.r.t. $l$ (see Lemma 7). An example where a face perpendicular to $l$ prevents $P$ from being castable although $l$ does not intersect any of the black regions induced by $P$ is shown in Fig. 4.

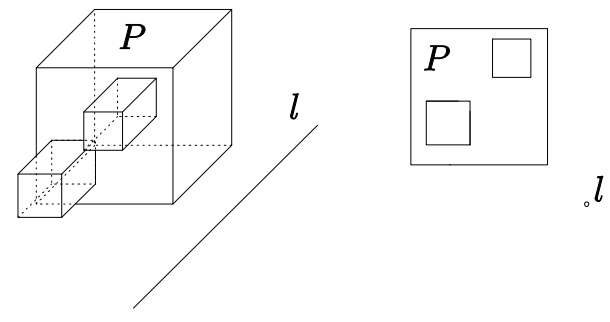

Fig. 4: Example of polyhedron $P$ that is not castable with respect to $l$ although $l$ does not intersect the black regions induced by $P$. Figure shows perspective view and front view.

Hence, Lemma 7 can be restated the following way:

Lemma 8. The polyhedron $P$ is castable w.r.t. $l$ if $l$ does not intersect any of the black regions induced by $P$ except possibly a far wedge of a reflex edge of $P$ and if every face of $P$ perpendicular to $l$ is removable w.r.t. $l$.

Computer-Aided Design \& Applications, Vol. 4, Nos. 1-4, 2007, pp 1-10 
Armed with this characterization of valid casting lines, to report all combinatorially distinct valid casting lines, we need to augment McKenna and O'Rourke's graph $G$ representing the arrangement of $O(n)$ lines arising from black regions.

Each node of the graph $G$ represents a distinct class of lines, which means that a line represented in one node cannot move to a line represented in another node without intersecting one of the $O(n)$ lines in $A$.

There are two steps to the augmentation since it needs to account for lines perpendicular to faces and edges of $P$. For the edges of $P$, we simply refine $G$ by adding nodes that represent lines perpendicular to edges of $P$. Three arbitrary skew lines represent a path in $G$. For each triple of skew lines, we find all the $O(n)$ lines that touch these three skew lines and are perpendicular to an edge of $P$. Each such line, along with the three skew lines will form a new node of $G$. We insert these nodes according to their order of appearance on the path, therefore it takes $O(n \log n)$ time to insert $O(n)$ new nodes. Since there are $O\left(n^{3}\right)$ triples of skew lines, the total time is $O\left(n^{4} \log n\right)$. Once this augmentation is complete, any line perpendicular to a reflex edge of $P$ will be represented in a node of $G$.

For lines perpendicular to faces, we build a secondary structure in the following way. For each set of faces of $P$ with parallel normals, we construct an arrangement on a parallel plane $\pi$ by projecting the $O(n)$ lines bounding the black regions onto $\pi$. The arrangement has $O\left(n^{2}\right)$ complexity. Each cell of the arrangement is labeled as either valid or invalid using a standard traversal. We also preprocess the arrangement for point location. Now, given a line $l$ perpendicular to a face, we can determine in $O(\log n)$ time which cell of the arrangement contains the point $\pi \cap l$, thereby determining the validity of $l$. This yields the following theorem.

Theorem 4. Given a polyhedron $P$ with combinatorial complexity $n$, it is possible to report all of the valid casting lines for $P$ in $\mathbb{R}^{3}$ in time $O\left(n^{4} \log n\right)$.

\subsection{Polyhedra with $\Omega\left(n^{4}\right)$ Combinatorially Distinct Valid Casting Lines}

In this section, we examine the complexity of all the combinatorially distinct valid (robust) casting lines for $P$ and show that there exists a class of polyhedra with $\Omega\left(n^{4}\right)$ combinatorially distinct valid (robust) casting lines. This implies that all of the algorithms presented above are almost worst case optimal. We now outline the construction of the lower bound.

We construct two cylinders whose cross section is a simple polygon $S$ with $r$ vertices with the property that there exist $\Omega\left(r^{2}\right)$ combinatorially distinct valid centers of rotations if two dimensional clamshell casting is considered. The lower bound example in two dimensions was published by Bose et al. [5].

The polyhedron $P$ consists of $n=4 r+8$ vertices. The two cylinders with $2 r$ vertices each are joined by a small cylinder whose cross section is a square. The first cylinder $C_{1}$ is located between the two planes $z=0$ and $z=1$. The second cylinder $C_{2}$ is located between the two planes $z=2$ and $z=3$. The two cylinders $C_{1}$ and $C_{2}$ are joined by an axis-parallel box with the corners $\left(-\frac{3}{4}, 0,1\right),\left(-\frac{9}{16}, \frac{3}{16}, 2\right)$. For an illustration of $P$, refer to Fig. 5 .

The black regions induced by the faces and reflex edges of the box joining the two cylinders $C_{1}$ and $C_{2}$ are completely located in $x=d_{1}, d_{1} \in\left[-\frac{3}{4},-\frac{9}{16}\right]$ and $y=d_{2}, d_{2} \in\left[0, \frac{3}{16}\right]$. Any line passing through one of the $\Omega\left(r^{2}\right)$ bounded valid regions induced by $C_{1}$ and passing through one of the $\Omega\left(r^{2}\right)$ bounded valid regions induced by $C_{2}$ is a valid casting line for $P$. Hence, there exist $\Omega\left(r^{4}\right)$ combinatorially distinct valid casting lines for $P$. Since none of the bounded valid regions induced by $C_{1}$ and $C_{2}$ restricts a line passing through the valid region to be perpendicular to a face of $P$, there are also $\Omega\left(r^{4}\right)$ combinatorially distinct valid robust casting lines for $P$. Hence, there exist $\Omega\left(n^{4}\right)$ combinatorially distinct valid (robust) casting lines for $P$.

Computer-Aided Design \& Applications, Vol. 4, Nos. 1-4, 2007, pp 1-10 


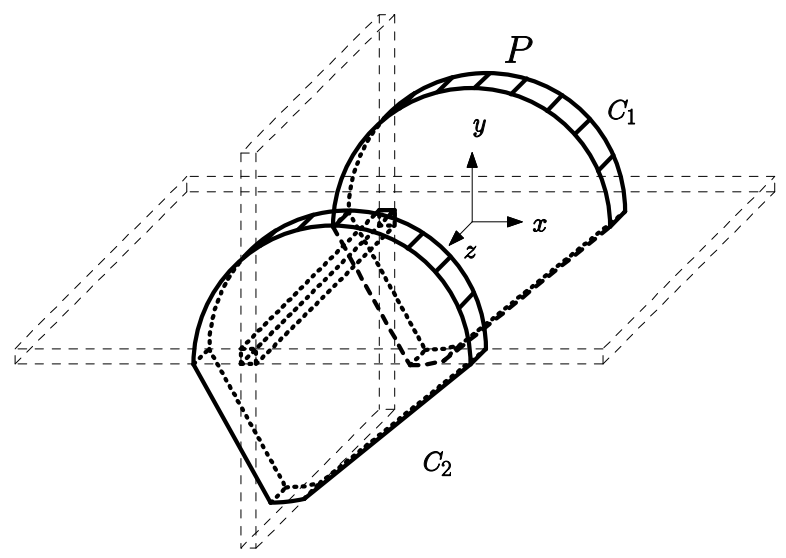

Fig. 5: Example of a polyhedron with $\Omega\left(n^{4}\right)$ combinatorially distinct valid casting lines is shown in bold. The two sets of planes containing all of the black regions introduced by the box joining $C_{1}$ and $C_{2}$ are shown as dashed boxes.

\section{CONCLUSION AND FUTURE WORK}

We have initiated the study of clamshell casting in three dimensions. We developed algorithms to test if a given line is a valid casting line for a given polyhedron and algorithms to report all of the valid casting lines for a given polyhedron. The running times of the algorithms are shown to be almost worst case optimal if the aim is to report all of the combinatorially distinct valid casting lines. The following interesting related problems require further research.

1. We only test whether the cast of an object with piecewise linear boundary can be opened by any angle without breaking the object or the cast. However, it remains to test whether the cast can be opened by a sufficiently large angle to remove the object from the cast without breaking the object or the cast.

2. The boundary of the object is defined to be the cast. However, the thickness of the cast is not neglectable. Hence, the cast needs to be assigned a thickness.

3. Since many objects do not have a piecewise linear boundary, the algorithms should be extended to handle more general object boundaries.

\section{REFERENCES}

[1] Ahn, H.; de Berg, M.; Bose, P.; Cheng, S.; Halperin, D.; Matousek, J.; Schwarzkopf, O.: Separating an object from its cast, Computer-Aided Design, 34, 2002, 547-559.

[2] Bose, P.: Geometric and computational aspects of manufacturing processes, Ph.D. Thesis, McGill University, Montreal, PQ, 1994. http://cg.scs.carleton.ca/ jit/publications/papers/b94.ps

[3] Bose, P.; Bremner, D.; van Kreveld, M.: Castability of simple polyhedra, Algorithmica: Special Issue on Manufacturing, 19, 1997, 84-113.

[4] Bose, P.; Morin, P.; Smid, M.; Wuhrer, S.: Rotational clamshell casting in three dimensions, TR-06-04, Carleton University, Ottawa, ON, 2006. http://cg.scs.carleton.ca/ swuhrer/Publications/index.html

[5] Bose, P.; Morin, P.; Smid, M.; Wuhrer, S.: Rotational clamshell casting in two dimensions, TR-06-03, Carleton University, Ottawa, ON, 2006. http://cg.scs.carleton.ca/ swuhrer/Publications/index.html

[6] Bose, P.; Toussaint, G.: Geometric and computational aspects of manufacturing processes. Computers and Graphics, 18(4), 1994, 487-497.

[7] Fekete, S.; Mitchell, J.: Terrain decomposition and layered manufacturing, International Journal of Computational Geometry, 11(6), 2001, 647-668.

[8] Goodman, J.; O’Rourke, J.: Handbook of Discrete and Computational Geometry, Second Edition, Chapman \& Hall CRC, 2004

[9] McKenna, M.; O'Rourke, J.: Arrangements of lines in 3-space: A data structure with applications, Proceedings of the fourth annual symposium on Computational Geometry, 1988, 371-380.

[10] Sack, J.; Urrutia, J.: Handbook of Computational Geometry, Elsevier, 2000.

[11] Stolfi, J.: Oriented Projective Geometry- A Framework for Geometric Computations, Academic Press Professional Inc, 1991.

Computer-Aided Design \& Applications, Vol. 4, Nos. 1-4, 2007, pp 1-10 